\title{
bioethics
}

\section{Personal genomes: no bad news?}

\begin{tabular}{|r|l|}
\hline Journal: & Bioethics \\
\hline Manuscript ID: & BIOT-1149-12-10-ART \\
\hline Manuscript Type: & Original Article \\
\hline Keywords: & $\begin{array}{l}\text { Human Genome Project, Personalisation, Genetic Determinism, } \\
\text { Privacy }\end{array}$ \\
\hline \multicolumn{2}{|l}{} \\
\hline
\end{tabular}

SCHOLARONE ${ }^{m}$

Manuscripts 


\title{
Editorial: Personal genomes: no bad news?
}

\section{Ruth Chadwick}

\section{Cardiff University}

\begin{abstract}
Reflecting on the history of Bioethics over the last twenty-five years it is impossible not to note the central place of genetics and genomics in bioethical discussions, even though this journal has not been dominated by them. A decade since the completion of the Human Genome Project, assessment is under way of what has been achieved.
\end{abstract} From some points of view, there may be disappointment, that having acquired so much information, the applications e.g. in clinical practice are less than hoped for, although it might be claimed that the 'bad news' stories have also been overdone. On the other hand some direct to consumer companies are marketing products that are, to say the least, troubling, purporting as they do to offer not only information 
about an individual's genome that is not only clearly health related but also advice on behavioural characteristics, the science of which is under developed. These facts have inspired a considerable amount of work on the sociology of promise and expectation. Technoscientific developments are accompanied by promises, to the public at large or to specific publics, to governments and to organisations. Related to promise is the concept of imaginary, an imagined future. Imaginaries are used by specific groups in the furtherance of other purposes, which can be political or economic. Imaginaries go beyond the thought experiment, a tool long used by philosophers to think through the possible implications and consequences of particular developments or courses of action, because they are used not just as an analytical tool but also to further particular purposes. An example might be the imaginaries that have been at work in the debates on genetically modified crops - e.g. 'feeding the world' is one imagined future; 'destroying biodiversity' is another. Clearly these imaginaries are likely not only to reflect particular commitments but also to have ethical implications, because the extent to which they are influential 
can affect the interests of individuals, groups and societies for good or ill.

\begin{abstract}
After ten years it is appropriate to take stock of the imaginaries that have been at work over the past period, and evaluate their success, or not, in an attempt to see the extent to which they are still at work at the present time. Ethicists, bioethicists in particular, are often accused, if not of attacking straw men, of considering possible consequences at too early a stage of development, or again, of coming in too late.
\end{abstract}

In the early days of the debates on genetics there was considerable anxiety about genetic discrimination, whether by insurance companies or employers. The relevant negative imaginary was 'creation of a genetic underclass', or 'a genetically segregated society'. On the other hand one positive (from some points of view) imagined future, contained for example in the 2003 report of the UK Department of Health, OurInheritance Our Future, involved that of health and 
lifestyle advice 'tailored to the individual'. Others, even those who regarded the future employment of genetic information for health care purposes as potentially positive, were cautious about what they saw here as a 'boutique model' of health care, and instead advocated an imagined future in which genetic information could be a mechanism for the reduction of inequities in access to health care between the developed and developing world.

\begin{abstract}
At this point in time we find ourselves at a period when the science is once more rapidly developing, the prospect of relatively inexpensive and rapid whole genome sequencing of individuals on the horizon.

From the time and expense involved in producing the first human genome sequence, the science has come a long way. It is appropriate also to reflect on the extent to which there have also been developments in ethics over the last ten years,
\end{abstract}

It is not necessary to subscribe to full-blown genetic exceptionalism to take the view that there have been developments in ethical thinking 
alongside the developments in genomics and in the social and political context in which these have taken place. In the early nineties the issues surrounding disclosure and rights to know and not to know tended to fall into two main types: conflicts of interest in the genetic counselling setting, where one person's reluctance to disclose information might have implications for another's right to know it; and situations where an individual had to deal with predictive information, concerning which they would prefer not to have knowledge. So discussion turned on the circumstances in which it could be right to disclose; and choice in relation to tests. The ethical considerations at stake included, primarily, individual autonomy, confidentiality, and beneficence. In 1997 for example, Jørgen Husted drew attention to two conceptions of autonomy, a 'thin' one which was typically used to support the benefits of genetic information; and a 'thick' one which supported a right not to know.

The arguments of the time are still rehearsed in different contexts, but I want to draw attention to two trends since that time. The first is a 
move in ethics towards more public health considerations: the other is towards a greater emphasis on principles of solidarity and equity. Thus the HUGO Statement (2007) on Pharmacogenomics, Solidarity and Equity states:

"the Committee considers it urgent that the ethical principles of solidarity and equity be given increased attention

- Solidarity: Because of shared vulnerabilities, people have common interests and moral responsibilities to each other. Willingness to share information and to participate in research is a praiseworthy contribution to society.

- Equity: To reduce health inequalities between different populations, and to work towards equal access to care is an important prerequisite for implementing genomic knowledge for the benefit of society."

The emphasis is thus on information sharing rather than on greater protection of individual rights.

In the light of the fast pace of development of the technologies ethical thinking is faced with the move towards greater 'personalisation', on 
the one hand, and more global developments, on the other, in relation both to the facilitating of international biobanks (requiring thinking about harmonisation of standards and ethics) and to claims for sharing the benefits that genomics has to offer on a global scale. The discussion of rights to know and not to know has to be continually renegotiated in the light of these interests in tension.

One of the principal tasks of the bioethical endeavour is to map the ways in which the boundaries of key concepts shift or become blurred over time. For example, in the context of reproductive ethics, this has been the case in relation to concepts such as 'parent'. I want to focus in this short editorial essay on three concepts : the concept of personalisation, genetic determinism, and privacy.

Personal

What is meant by 'personal' in the personal genome? Let us first consider the related concept of 'personalisation'. Arguably the majority of discussion about pesonalisation has taken place in relation to pharmaceuticals, although nutrigenomics has not been far behind. 
In the case of pharmacogenomics, in practice it might mean no more than doing a genetic test to assign a person to one group or another - a poor or good responder to a drug, for example. If it is just allocation to a group, however, to use the term 'personalisation' is, to say the least, potentially misleading. The relevant difference is between this patient and the whole population, rather than between this patient and membership of any given group.

Personalisation can mean, secondly, particular action on the basis of genetic information - the choice between drug A and drug B; the selection of the appropriate dosage; the choice between drug A and no medication at all. In this sense, however, it could be argued that medicine has always been personalised in the sense of the application of professional judgment to the individual case. In this sense, again, to personalise and to individualise appear to be equivalent. In a third sense to 'personalise' might mean to treat the patient as a person, where a person is understood as a self-aware being with their own goals and life plans. It is in this sense that complementary therapies 
are sometimes said to be more concerned with the person that just with the symptoms of the individual case. This is where a difference between individualisation and personalisation may become apparent. To treat a patient as a person, rather than just as one (individual) case, is to have regard to their goals as well as symptoms.

The sense in which personalisation is marketed as 'tailoring', however, seems not to be precisely equivalent to any of these meanings, although they are all significant from an ethical point of view. In order to have full tailoring to this one individual/person before you, arguably that person's whole genome needs to be looked at. While the prospect of whole genome sequencing is now on the agenda for debate the extent to which it is possible to make sense of all the information in a person's genome, however, is still a long way off.
A relevant sense in which this personalisation has ethical implications, however, is that such personal genomics is identifying 
If it becomes impossible to conceal one's identity (e.g against a totalitarian regume) and key facts about oneself, this raises issues about both genetic determinism and privacy.

\title{
Genetic determinism
}

In the immediate aftermath of the Human Genome Project, it was claimed that genetic determinism was dead. This was due to the discovery that there were far fewer genes in the human genome than had been expected, around 24.000 rather than 100,000. Surely, such a small number of genes could not account for the complexity of the beings that we are. This raises the question of the level at which the number becomes too small to do that. If there is determinism, it must lie elsewhere than in the genes per se.

\author{
Of course, there had been discussions of genetic determinism that \\ predated this discovery. There had been debate about the difference
}


between the ways in which a gene can influence rather than determine. Even in the case of a single gene disorder, there is variation in penetrance and severity. It is also important to remember that these discussions of determinism relate to the nuclear genome: disorders in the mitochondrial genome, inherited down the female line, produce very variable results. Since the Human Genome Project much more is also understood about such things as copy number variation and epigenetics.

With the advent of whole genome sequencing, however, genetic determinism is back on the agenda, and is heavily implicated in an imagined future in which it is possible to predict people's future talents and behavioural traits and use these predictions to inform training and educational needs. We might call this imaginary genetically informed education.

To return to the relevant conceptual question for Bioethics, is this reemerging genetic determinism, determinism in the same sense as the 
genetic determinism that was rejected at the turn of the century? I think not. It seems to me that what was being rejected in that debate was the 'gene for $\mathrm{x}$ ' model that has also been implicated in various imaginaries, positive and negative.

The genetic determinism that is emerging now is that the genome in all its complexity, is deterministic, taking into account the volume of data that can be made available on the precise sequence in an individual's genome, including all the myriad ways in which he or she differs from other individuals.

So we need to be clear about the form that genetic determinism is now taking, in addition to the ethical implications of its employment. It is still counteracted by other considerations. One of these recently discussed in the media is the 'ten thousand hours' hypothesis that anyone can develop a talent for $\mathrm{x}$ if he or she devotes ten thousand hours to x. It is not necessary for some genetically based predisposition to be present. Again, genetic determinisn is countered 
by arguments about gene-environment interaction. Such interaction, however, has to take into account not only the environment external to the body, which can be both physical and social, but also the environment internal to the body, and in so far as that involves interaction between different genetic factors it arguably supports a view that the whole genome is deterministic rather than any given part of it.

\section{Privacy}

The new generation of sequencing technologies massively increases the density of analyses that can be performed in a single run, as compared with conventional sequencing. But what is meant by 'whole genome' here - all the coding exons in the genome or perhaps more usefully a subset of these we are interested in for diagnostic purposes?

While for the near future targeted investigations are likely to remain necessary, the prospect of whole genome sequencing changes the scope of what is talked about in the right to know and right not to 
know debate. While there have always been issues concerning disclosure of incidental findings (e.g. non-paternity), the possibilities of whole genome sequencing open up the prospect of knowing everything in the genome. The very concept of 'incidental findings' becomes redundant. There is of course a question about the extent to which the volume of information will be meaningful, at least in the early stages of this development, but are there grounds for thinking that the privacy concerns are increased? If there is concern about access to knowledge about one's genome, does the volume of information that may be accessed give rise to a parallel increase in the concerns about privacy?

These may be concerns influenced by a form of genetic determinism, that to know someone's genome is to know everything about the person. Again there are different imagined futures here. One is of a genetic surveillance society (encouraged by, for example, by developments such as the UK National DNA database). At the opposite extreme is of an open source genetic society, where 
individuals are free and open with each other about their genomes, as instanced, for example, by the Personal Genome Project.

It seems appropriate to engage in this particular discussion with reference to developments taking place in other contexts. There is evidence that in relation to the internet, for example, the increasing popularity of Facebook, Twitter and blogs provides evidence of changing attitudes towards privacy. It is, then, at least of interest to ask why in relation to genetics, in part6icular, has privacy been such a central concern. Different explanations are possible, and are not necessarily mutually exclusive. There may be generational differences; organisations and groups may be successful in deploying their imaginaries in different contexts.

\begin{abstract}
Different ways to counter anxieties are available. One is to try to allay concerns about privacy. The advent of 'celebrity genomes' are one instance of this: where key figures willingly publish their genomes to
\end{abstract}




\begin{abstract}
illustrate a different attitude towards privacy and disclosure, but such plans have not been met without some alarm.
\end{abstract}

The second, not unrelated. trend is towards rethinking the concept and value of privacy. In the surveillance different context, it has been claimed that even if we could rely on public institutions to adhere completely to regulations on data protection, there is still a need to look at the issues in different ways.. In the genomics context, there has been a suggestion that emphasis on privacy cannot be guaranteed any longer and should be replaced by the concept of open consent. The concept of open consent is used in the context of the Personal Genome Project, which aims to build a framework for the development and evaluation of personal genome technologies. Towards this end, open consent implies that research participants accept that their data may be included in an open access database with no guarantees of privacy and confidentiality. 


\title{
Towards the future
}

\begin{abstract}
Although a great deal of bioethical discussion has taken place in relation to genomics, arguably the most interesting phase is yet to come, and it is to be hoped that debate in this journal will play a significant part in the debates about genomic futures.
\end{abstract}

